\title{
Current symptom-based risk scores for stable coronary artery disease evaluation are not applicable in COPD patients
}

\author{
Christoph Beyer ${ }^{1}$, Alex Pizzini (10², Anna Boehm (1) ${ }^{2}$, Judith Loeffler-Ragg ${ }^{2}$, \\ Guenter Weiss ${ }^{2}$, Gudrun Feuchtner ${ }^{3}$, Axel Bauer ${ }^{1}$, Guy Friedrich ${ }^{1}$ and \\ Fabian Plank (10 1
}

Affiliations: 'Dept of Internal Medicine III - Cardiology and Angiology, Medical University Innsbruck, Innsbruck, Austria. ${ }^{2}$ Dept of Internal Medicine II - Infectious Diseases, Pneumology and Rheumatology, Medical University Innsbruck, Innsbruck, Austria. ${ }^{3}$ Dept of Radiology, Medical University Innsbruck, Innsbruck, Austria.

Correspondence: Fabian Plank, Dept of Internal Medicine III - Cardiology and Angiology, Medical University Innsbruck, Anichstraße 35, A-6020 Innsbruck, Austria. E-mail address: fabian.plank@i-med.ac.at

\section{ABSTRACT}

Background: Cardiovascular diseases are arguably the most important comorbidity in patients with COPD. Despite an increased prevalence of coronary artery disease (CAD) in COPD patients, there are no dedicated diagnostic recommendations.

Objectives: We investigated whether COPD patients receive adequate primary evaluation of CAD despite overlapping symptoms.

Methods: In total, 302 patients with COPD who underwent invasive coronary angiography (ICA) were retrospectively matched (for age, body mass index and cardiovascular risk factors) with 302 patients without functional lung diseases. Quality and onset of symptoms prior to ICA were documented, and individual patients' pretest probabilities according to European Society of Cardiology (ESC) guidelines were calculated. Endpoints were delay of ICA referral after symptom onset and clinical outcome, defined as subsequent revascularisation.

Results: Mean delay between symptom onset and ICA was $19.9 \pm 22.0$ months in COPD patients compared to $8.3 \pm 12.7$ months in the control group $(\mathrm{p}<0.0001)$. COPD patients had a lower rate of typical chest pain ( $25.2 \%$ versus $38.1 \%, \mathrm{p}=0.0009$ ), and dyspnoea only $(18.2 \%$ versus $26.8 \%, \mathrm{p}=0.015)$. Sub-analysis of Global Initiative for Chronic Obstructive Lung Disease (GOLD) grades revealed an incremental delay with increasing COPD severity: GOLD 1: $16.1 \pm 17.3$ months; GOLD 2: $17.6 \pm 22.1$ months; GOLD 3: 20.1 \pm 21.3 months; and GOLD 4: $24.2 \pm 23.4$ months. Overall significant CAD prevalence ( $>70 \%$ stenosis) was $35.3 \%$; the revascularisation rate increased with higher pretest probability for the control group but decreased for patients with COPD GOLD 1-4.

Conclusion: Patients with COPD are insufficiently evaluated for CAD due to overlapping symptoms. Current CAD risk scores for stable chest pain appear inappropriate for patients with COPD.

@ERSpublications

Coronary artery disease evaluation is delayed in COPD patients compared to patients without obstructive lung disease. Due to overlapping symptoms, pretest probabilities of current stable angina models do not fit patients with COPD. https://bit.ly/318RkNz

Cite this article as: Beyer C, Pizzini A, Boehm A, et al. Current symptom-based risk scores for stable coronary artery disease evaluation are not applicable in COPD patients. ERJ Open Res 2020; 6: 00492-2020 [https://doi.org/10.1183/23120541.00492-2020].

Received: 13 July 2020 | Accepted after revision: 27 July 2020

Copyright $\odot$ ERS 2020. This article is open access and distributed under the terms of the Creative Commons Attribution Non-Commercial Licence 4.0 


\section{Introduction}

COPD is a common, preventable and treatable disease with persistent respiratory symptoms that affects an estimated number of 328 million people worldwide [1]. Besides improvements in sanitation, available respiratory medication and overall life condition, absolute mortality is expected to increase with an annual rate of at least 3.2 million people [2].

In addition to increased primary prevention of COPD, the clinical management of patients continues to improve beyond the respiratory system in order to counteract this trend.

A high number of concomitant morbidities have been identified, attributed both to COPD's disease-related systemic effects and to similar predisposing risk factors [3,4]. Among all comorbidities, coronary artery disease (CAD) is not only the leading cause of mortality worldwide, but also the main cause of death in COPD patients $[5,6]$. A recent meta-analysis reported a two- to five-fold increase of ischaemic heart disease in patients with COPD compared to the general population [7].

Although the importance of this specific comorbidity is increasingly recognised, there is a significant lack of dedicated guidelines in both screening and managing CAD in COPD patients. Similar clinical presentation of both diseases including reduced exercise ability, dyspnoea and chest discomfort are complicating the discrimination between the two entities. This may lead to a "first-come-first-serve" principle, while neglecting the other disease.

The aim of our study was to evaluate whether patients with COPD are correctly assessed for their CAD risk and referred in a timely manner to ICA or whether there are disadvantages in the process of primary CAD evaluation compared to the general population.

\section{Materials and methods}

\section{Study population}

This retrospective matched cohort study included patients with known COPD of any degree [8] who underwent elective invasive coronary angiography (ICA) for primary evaluation of suspected CAD at a tertiary care hospital, between 2000 and 2019. All patients were referred on the basis of clinical presentation with chest pain and/or dyspnoea and a presumption of cardiac aetiology. The study was approved by our local institutional review board, and patients' informed consent forms were waived.

Patients were included if COPD was diagnosed according to the Global Initiative for Chronic Obstructive Lung Disease (GOLD) guideline, presenting typical symptoms and a post-bronchodilator fixed ratio of forced expiratory volume in $1 \mathrm{~s}\left(\mathrm{FEV}_{1}\right)$ versus forced vital capacity $<0.7$ [8]. A patient population matched control group without medical history of COPD was created using a local ICA registry.

Non-symptomatic COPD patients with ICA examinations for CAD screening and patients undergoing acute ICA due to acute coronary syndromes were excluded. Patients with previously known CAD, prior ICA and revascularisation (including percutaneous coronary intervention and coronary artery bypass grafting $(\mathrm{CABG})$ ) were further excluded from our analysis. A flowchart depicting patient selection is presented in figure 1.

\section{Symptom definition}

The analysis of symptoms that triggered referral for invasive coronary evaluation included both intensity and presentation of chest pain and dyspnoea. Chest pain was classified into typical angina, atypical angina and non-anginal as recommended by the European Society of Cardiology (ESC) [9] and further quantified using the Canadian Cardiovascular Society score (CCS grade 1 to 4) for chest pain [10]. In case of absent chest pain, dyspnoea was used as the primary symptom for the CAD risk assessment according to the 2019 ESC recommendations and was further classified using the New York Heart Association functional classification (NYHA grade 1 to 4 ) [11].

\section{Pretest probability of obstructive CAD}

Patients' clinical likelihood of obstructive CAD was calculated using the modified Diamond and Forrester model from the current ESC 2019 guidelines. This predictive model estimates the pretest probability of obstructive CAD based on age, sex and presented symptoms (typical chest pain, atypical chest pain, non-anginal chest pain, dyspnoea) [9].

\section{Data collection}

Data on onset, quality and intensity of patients' symptoms were retrospectively collected using a clinical documentation system based on routinely conducted specific anamnestic questionnaires.

Symptoms were only considered if a definite onset was documented and the symptoms were consistently reported and persisted over subsequent documentations. 


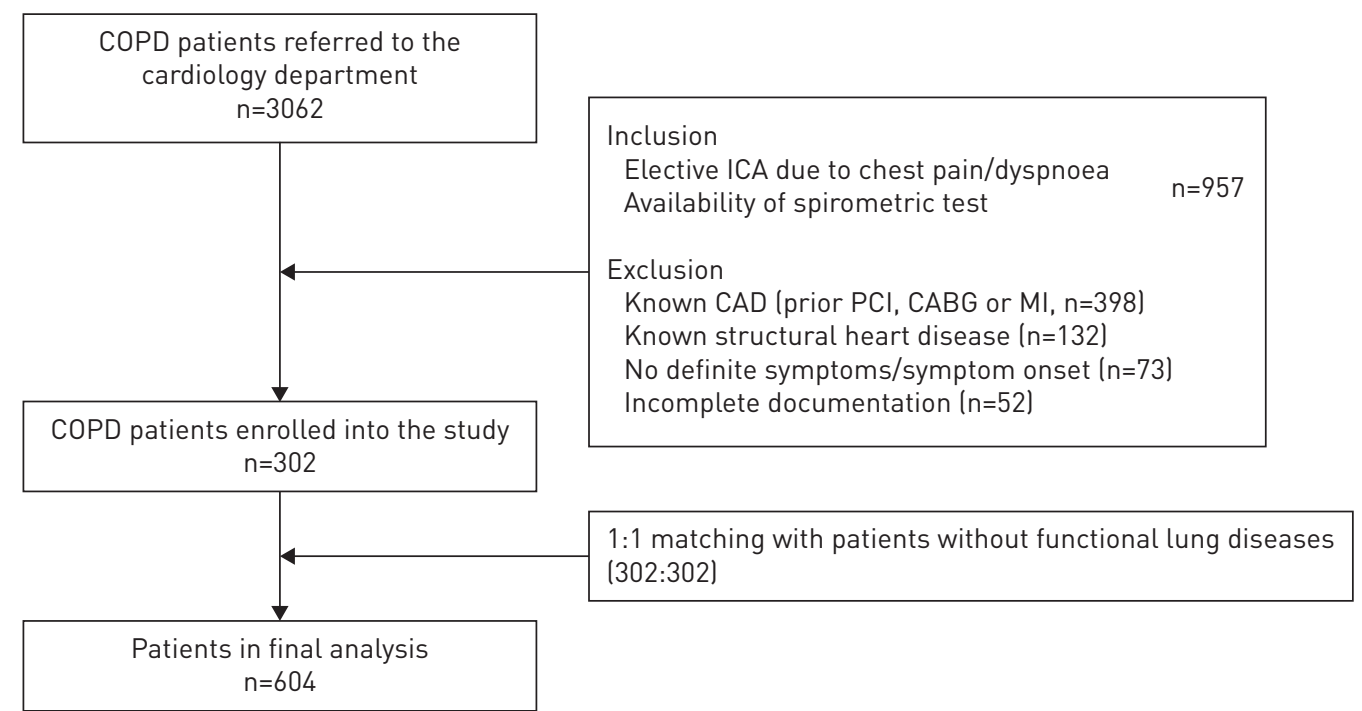

FIGURE 1 Flowchart depicting patient inclusion. CABG: coronary artery bypass graft; CAD: coronary artery disease; ICA: invasive coronary angiography; $\mathrm{MI}$ : myocardial infarction; $\mathrm{PCl}$ : percutaneous coronary intervention.

Furthermore, patients' cardiovascular risk factors, pulmonary function parameters and medication at the time of ICA were collected.

\section{Propensity score matching}

The baseline COPD study population was propensity score matched with patients from a local ICA registry of patients without airflow limitation with clearly defined symptom onset. Matching was performed with a priori determined factors using logistic regression (caliper 0.2 ) and a nearest-neighbour approach for age, male sex, hypertension, hyperlipidaemia, diabetes mellitus, family history of premature cardiac death, smoking and body mass index (BMI), as outlined in table 1.

\section{Endpoints}

The primary endpoint was the time interval between symptom onset and ICA. ICA results and revascularisation rate were also considered in the analysis for both cohorts.

The secondary endpoints were the diagnostic agreement of the ESC pretest probability calculation and the subsequent ICA findings [9].

The third endpoint was clinical follow-up, defined as major adverse cardiac events (according to the 4th universal definition [12]), cardiovascular and all-cause death.

\section{Statistical analysis}

Statistical testing was performed using SSPSTM software (V24.0; SPSS Inc., Chicago, IL, USA). Quantitative variables are expressed as mean \pm SD. In case of normal distribution, further comparisons were made using $t$-test and one-way ANOVA. Categorical variables are given as absolute values and percentages and were compared with Chi-squared and Fisher's exact test as appropriate. Spearman's rank correlation was used to test for a trend between COPD groups. A p-value of $<0.05$ was considered statistically significant.

\section{Results}

The final analysis included 604 patients, 302 with COPD (85 female, 28.2\%) and 302 matched patients without medical history of pulmonary disease (98 female, $32.5 \%$ ). After propensity score matching, there were no differences in age, body mass index, relative distribution according to gender, hypertension, dyslipidaemia, diabetes, family history of coronary heart disease or smoking. Of 302 patients with COPD, 34 (11.3\%) were classified GOLD 1, 120 (39.7\%) GOLD 2, 98 (32.5\%) GOLD 3 and 50 (16.6\%) GOLD 4. Patient characteristics are depicted in table 1.

The mean delay between the first documentation of symptoms and invasive angiography was $19.9 \pm 22.0$ months for patients with COPD and $8.3 \pm 12.7$ months for the control group $(\mathrm{p}<0.0001)$. Sub-analysing GOLD grades showed an incremental delay with increasing COPD severity: GOLD 1: 


\begin{tabular}{|c|c|c|c|}
\hline & Control & COPD & p-value \\
\hline Subjects $\mathrm{n}$ & 302 & 302 & \\
\hline Female & 98 (32.5) & 85 (28.2) & 0.29 \\
\hline Age years & $66.2 \pm 8.2$ & $66.3 \pm 8.2$ & 0.99 \\
\hline BMI & $27.7 \pm 4.4$ & $26.7 \pm 6.0$ & 0.29 \\
\hline Arterial hypertension & $239(79.1)$ & $231(76.5)$ & 0.49 \\
\hline Dyslipidaemia & 185 (61.3) & 185 (61.3) & 1.00 \\
\hline Diabetes & $50(16.6)$ & $51(16.9)$ & 1.00 \\
\hline Smoking & $258(85.4)$ & 259 (85.8) & 1.00 \\
\hline Family history of CAD & 45 (14.9) & 45 (14.9) & 1.00 \\
\hline PAD & $22(7.3)$ & $14(4.6)$ & 0.22 \\
\hline CKD $(\geqslant 3 b)$ & 4 (1.3) & $6(2.0)$ & 0.75 \\
\hline ASS & $180(59.6)$ & $198(65.6)$ & 0.19 \\
\hline Statin & $150(49.7)$ & $139(46.0)$ & 0.33 \\
\hline Ezetimibe & $6(2.0)$ & $6(2.0)$ & 1.00 \\
\hline B-blocker & 89 (29.5) & 93 (30.8) & 0.81 \\
\hline ACE inhibitor & 76 (25.2) & $97(32.1)$ & 0.07 \\
\hline AT2 channel blocker & 71 (23.5) & 59 (19.5) & 0.20 \\
\hline Calcium channel blocker & $46(15.2)$ & $44(14.6)$ & 0.82 \\
\hline Diuretic & 82 (27.2) & 98 (32.5) & 0.18 \\
\hline COPD (GOLD) 1 & & 34 (11.3) & \\
\hline COPD (GOLD) 2 & & 120 (39.7) & \\
\hline COPD (GOLD) 3 & & 98 (32.5) & \\
\hline COPD (GOLD) 4 & & $50(16.6)$ & \\
\hline
\end{tabular}

Data are presented as $\mathrm{n}(\%)$ or mean $\pm \mathrm{SD}$, unless otherwise stated. BMI: body mass index; CAD: coronary artery disease; PAD: peripheral artery disease; CKD: chronic kidney disease; ASS: aspirin salicylic acid; ACE: angiotensin-converting enzyme; GOLD: Global Initiative for Chronic Obstructive Lung Disease.

16.1 \pm 17.3 months; GOLD 2: $17.6 \pm 22.1$ months; GOLD 3: $20.1 \pm 21.3$ months; and GOLD 4: $24.2 \pm 23.4$ months ( $\mathrm{p}<0.001$ for trend) (table 2).

In total, 73 patients (12.1\%) were referred from the same hospital, 133 (22.0\%) from secondary care centres and 398 (65.9\%) from primary care physicians. The proportion of control and COPD patients did not differ between the referring institutions $(\mathrm{p}=0.24)$. Also, the mean delay between symptom onset and ICA between the respective groups (control, GOLD 1-4) was independent of referring physician $(\mathrm{p}=0.30)$.

ICA findings were comparable between the groups. While there was a higher prevalence of high-grade stenosis with more frequent subsequent percutaneous coronary intervention in the control group, overall revascularisation rate did not differ significantly between both patient groups (table 2).

For comparison of the CAD pretest probabilities (modified Diamond-Forrester), we further stratified the COPD patients into their respective GOLD stage. Overall, we found an intermediate mean pretest probability (control 24.1\%, COPD GOLD 1: 30.5\%, GOLD 2: $25.2 \%$, GOLD 3: $24.9 \%$ and GOLD 4: $32.2 \%)$.

We then compared the pretest probabilities in each group (control, GOLD 1-4) divided into obstructive $\mathrm{CAD}$ requiring revascularisation and negative findings. In patients without $\mathrm{COPD}$, the mean $\mathrm{CAD}$ pretest probability was significantly higher in obstructive CAD compared to negative findings. However, the pretest probability in patients with GOLD 1-4 did not differ between obstructive CAD and negative findings (figure 2). The overall pretest probability for COPD GOLD 4 was higher, based on a higher rate of symptoms. However, the predictive separation into obstructive CAD and negative findings was not found. This separation in the control group is attributable to a different rate in typical chest pain of CAD negative (19.7\%) and positive (40.4\%).

A mean follow-up of 3.2 years was available for all patients without differences between the groups $(\mathrm{p}=0.94)$. Overall, 22 patients $(3.6 \%)$ suffered a major adverse cardiac event, with a significantly higher rate in the COPD cohort $(6.0 \%)$ compared to the control group $(1.3 \%, \mathrm{p}=0.004)$. The cardiovascular mortality was significantly higher in the COPD group ( $3.6 \%$ versus $0.6 \% ; \mathrm{p}=0.021)$. Similarly, COPD patients had a higher all-cause mortality $(5.3 \%$ versus $2.0 \%, \mathrm{p}=0.049)$ compared to the control group. 


\begin{tabular}{|c|c|c|c|}
\hline & Control & COPD & $p$-value \\
\hline Subjects $\mathrm{n}$ & 302 & 302 & \\
\hline Typical chest pain & $115(38.1)$ & $76(25.2)$ & 0.0009 \\
\hline Atypical chest pain & $43(14.2)$ & 78 (25.8) & 0.0005 \\
\hline Non-anginal chest pain & $63(20.9)$ & 93 (30.8) & 0.0069 \\
\hline Dyspnoea only & $81(26.8)$ & 55 (18.2) & 0.015 \\
\hline \multicolumn{4}{|l|}{$\operatorname{ccs}$} \\
\hline 0 & $81(26.8)$ & 55 (18.2) & 0.015 \\
\hline 1 & $56(18.5)$ & $9(3.0)$ & $<0.0001$ \\
\hline 2 & $70(23.2)$ & $67(22.2)$ & 0.85 \\
\hline 3 & 34 (11.3) & $81(26.8)$ & $<0.0001$ \\
\hline 4 & $61(20.2)$ & 90 (29.8) & 0.0084 \\
\hline \multicolumn{4}{|l|}{ NYHA } \\
\hline 1 & $129(42.7)$ & 60 (19.9) & $<0.0001$ \\
\hline 2 & $139(46.0)$ & 93 (30.8) & 0.0002 \\
\hline 3 & 28 (9.3) & $121(40.1)$ & $<0.0001$ \\
\hline 4 & $6(2.0)$ & 28 (9.3) & $<0.0001$ \\
\hline Delay between symptoms and ICA months & $8.3 \pm 12.7$ & $19.9 \pm 22.0$ & $<0.0001$ \\
\hline \multicolumn{4}{|l|}{ ICA } \\
\hline Negative (no stenosis) & $128(42.4)$ & $139(46.0)$ & 0.41 \\
\hline$<50 \%$ stenosis & $14(4.6)$ & $24(8.0)$ & 0.13 \\
\hline $50-70 \%$ stenosis & $36(11.9)$ & $50(16.6)$ & 0.13 \\
\hline$>70 \%$ stenosis & $113(37.4)$ & $79(26.2)$ & 0.0039 \\
\hline $100 \%$ stenosis & $11(3.6)$ & 10 (3.3) & 1.00 \\
\hline Revascularisation (PCI, CABG) & $118(39.1)$ & $94(31.1)$ & 0.050 \\
\hline Follow-up months & $38.4 \pm 39.5$ & $38.4 \pm 39.0$ & 0.94 \\
\hline MACE & $4(1.3)$ & $18(6.0)$ & 0.004 \\
\hline Cardiovascular death & $2(0.7)$ & $11(3.6)$ & 0.02 \\
\hline All-cause death & $6(2.0)$ & $16(5.3)$ & 0.05 \\
\hline
\end{tabular}

\section{Discussion}

The present analysis showed a significant delay in invasive CAD evaluation of patients with COPD compared to subjects without airflow limitation. Nevertheless, both cohorts had comparable ICA findings and subsequent revascularisation rates. Moreover, the established CAD pretest probability score was not applicable in COPD patients due to a profound miscalculation of their respective CAD risk [9]. These results confirm our working hypothesis that COPD is an important risk factor that is currently neglected

FIGURE 2 Pretest probabilities according to modified DiamondForrester (ESC 2019) for control and COPD Global Initiative for Chronic Obstructive Lung Disease (GOLD) 1 to 4. NS: not significant.

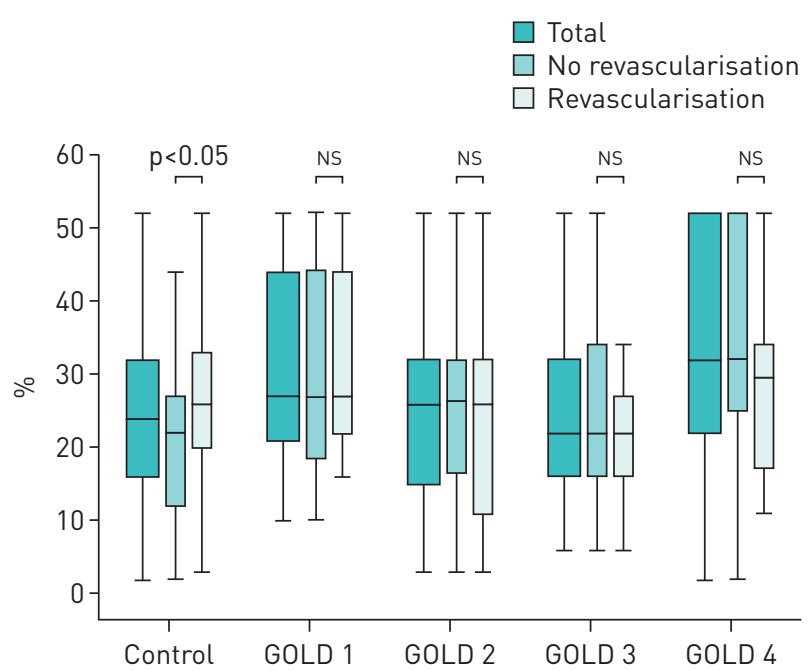


in the established guidelines for primary diagnostic evaluation of CAD. Even in medium-term follow-up, the present COPD cohort had a significantly higher cardiovascular and all-cause mortality compared to the control group. To the best of our knowledge, this is the first study to elucidate this marked systemic diagnostic deficit.

The awareness of the broad comorbidity spectrum in COPD patients has substantially increased in recent years [13]. In addition to a variety of shared risk factors, the increase in systemic inflammation markers and oxidative stress mediators lead to endothelial dysfunction and further promote comorbidities $[14,15]$.

Particularly, a direct influence of COPD on the progression of atherosclerosis has been reported [16], and thus COPD must be considered as a major independent risk factor for long-term cardiovascular mortality in patients with concomitant ischaemic heart disease [17]. On the other hand, COPD patients are at higher risk for insufficient evaluation and treatment of an accompanying cardiovascular disease. This is further substantiated by the significant difference in cardiovascular and overall mortality rates in our study.

The strong association between COPD and CAD warrants a refined approach in the diagnostic process, and although the GOLD guidelines recognise CAD as a major comorbidity, they do not recommend a different diagnostic path for patients with COPD [8]. Likewise, the recently updated ESC guidelines for chronic coronary syndromes do not provide any dedicated recommendations for COPD patients, despite their large number and their significantly higher risk for CAD $[2,7,9]$.

Primary CAD evaluation has always been based on a calculation of the clinical likelihood of obstructive CAD in order to provide physicians with guidance for further downstream testing $[9,17]$. The Diamond and Forrester model is the widest established calculator for the evaluation of pretest probability, based on age, sex and chest pain [18]. This model has been revised several times and was most recently extended by dyspnoea as an additional symptom $[9,19]$.

Despite these continuous amendments, this model only fits the general population but is not adjusted for specific comorbidities, particularly for patients with a symptom-overlapping pulmonary disease. This may lead to misinterpretation of the clinical presentation and the symptoms' origin and might further result in incorrect diagnostic steps. When entered into the modified Diamond and Forrester plot, particularly medium- to high-grade COPD patients showed a larger scatter of pretest probabilities in our analysis and an inferior correlation with subsequent revascularisations compared to non-COPD patients.

The initial underestimation and prolonged diagnostic evaluation of CAD in COPD patients stands in contrast to the general aspirations of the ESC. The recent guidelines put special emphasis on improving the low diagnostic yield of invasive coronary angiographies [20]. Therefore, the overall pretest probability threshold in the Diamond-Forrester model has been lowered to reduce unnecessary invasive examinations [9]. However, this development towards diagnostic restraint does not improve the considerations of COPD patients. The importance of an early CAD diagnosis in this patient population is reflected by a six times higher risk of hospitalisation in COPD patients with cardiovascular comorbidities compared to those without such comorbidities [21].

To overcome these difficulties in primary CAD evaluation in COPD patients, an increase in noninvasive evaluation is needed. Functional stress testing is often not feasible in patients due to exercise incapability, hence anatomical imaging would be superior as a noninvasive testing option. Besides a high negative predictive value regarding major adverse cardiac events, coronary computed tomography (CT) evaluation could be easily integrated into routine patient care [22]. Low-dose chest CT in patients with higher grade COPD is the gold standard for lung cancer screening. These routine exams could be modified to ECG-gated scans and - if indicated - to contrast-enhancement scans for further CAD evaluation. The ESC has recently upgraded coronary CT angiography as class level IB indication in patients with chronic stable coronary syndromes with low-to-intermediate risk of CAD [9].

Besides, even non-contrast scans would prove useful if evaluated for calcium score [23, 24]. This undervalued information from screening CTs has already proven predictive for mortality in a multicentre study. High calcium scores were associated with a three-fold increase of all-cause mortality in patients with COPD [25]. Therefore, standardised implementation of coronary CT could be a key tool to improve early CAD diagnosis in order to substantially reduce overall mortality rate in COPD patients.

Our study is limited by a retrospective data collection of the onset, type and severity of symptoms. However, the presented data show explicit results regarding the delay of ICA and clear symptomatic differences in COPD patients.

\section{Conclusion}

In conclusion, patients with $\mathrm{COPD}$ experienced a delay in $\mathrm{CAD}$ evaluation due to overlapping symptoms and insufficient consideration of their increased cardiovascular comorbidity. Current established CAD risk 
scores proved unsuitable for patients with COPD. Further diagnostic strategies must be developed to improve CAD diagnostics in this population.

Author contributions: C. Beyer, A. Pizzini, A. Boehm, J. Loeffler-Ragg, G. Weiss, G. Feuchtner, A. Bauer, G. Friedrich and F. Plank conceived and designed the study; C. Beyer and F. Plank drafted the manuscript; C. Beyer collected the data; C. Beyer, A. Pizzini, A. Boehm, J. Loeffler-Ragg, G. Weiss, G. Feuchtner, A. Bauer, G. Friedrich and F. Plank analysed and interpreted the data; and C. Beyer, A. Pizzini, A. Boehm, J. Loeffler-Ragg, G. Weiss, G. Feuchtner, A. Bauer, G. Friedrich and F. Plank revised and approved the manuscript

Conflict of interest: None declared.

\section{References}

1 Vos T, Flaxman AD, Naghavi M. Years lived with disability (YLDs) for 1160 sequelae of 289 diseases and injuries 1990-2010: a systematic analysis for the Global Burden of Disease Study 2010. Lancet 2012; 380: 2163-2196.

2 GBD 2015 Chronic Respiratory Disease Collaborators. Global, regional, and national deaths, prevalence, disability-adjusted life years, and years lived with disability for chronic obstructive pulmonary disease and asthma, 1990-2015: a systematic analysis for the Global Burden of Disease Study 2015. Lancet Respir Med 2017; 5: 691-706.

3 Cavaillès A, Brinchault-Rabin G, Dixmier A, et al. Comorbidities of COPD. Eur Respir Rev 2013; 22: 454-475.

4 Barnes PJ, Celli BR. Systemic manifestations and comorbidities of COPD. Eur Respir J 2009; 33: 1165-1185.

5 World Health Organization. The top 10 causes of death. 2016. www.who.int/news-room/fact-sheets/detail/ the-top-10-causes-of-death Date last updated: May 24, 2018; date last accessed: March 23, 2020.

6 Divo M, Cote C, de Torres JP, et al. Comorbidities and risk of mortality in patients with chronic obstructive pulmonary disease. Am J Respir Crit Care Med 2012; 186: 155-161.

7 Chen W, Thomas J, Sadatsafavi M, et al. Risk of cardiovascular comorbidity in patients with chronic obstructive pulmonary disease: a systematic review and meta-analysis. Lancet Respir Med 2015; 3: 631-639.

8 Vogelmeier CF, Criner GJ, Martinez FJ, et al. Global Strategy for the Diagnosis, Management, and Prevention of Chronic Obstructive Lung Disease 2017 Report: GOLD executive summary. Eur Respir J 2017; 49: 1700214.

9 Knuuti J, Wijns W, Saraste A, et al. 2019 ESC guidelines for the diagnosis and management of chronic coronary syndromes. Eur Heart J 2020; 41: 407-477.

10 Campeau L. Letter: Grading of angina pectoris. Circulation 1976; 54: 522-523.

11 The Criteria Committee of the New York Heart Association. Nomenclature and Criteria for Diagnosis of Diseases of the Heart and Great Vessels. 6th ed. Boston, MA, Little, Brown \& Co., 1994.

12 Thygesen K, Alpert JS, Jaffe AS, et al. Fourth universal definition of myocardial infarction (2018). Eur Heart J 2019; 40: 237-269.

13 Soriano JB, Visick GT, Muellerova H, et al. Patterns of comorbidities in newly diagnosed COPD and asthma in primary care. Chest 2005; 128: 2099-2107.

14 Fabbri LM, Luppi F, Beghé B, et al. Complex chronic comorbidities of COPD. Eur Respir J 2008; 31: 204-212.

15 Faner R, Gutiérrez-Sacristán A, Castro-Acosta A, et al. Molecular and clinical diseasome of comorbidities in exacerbated COPD patients. Eur Respir J 2015; 46: 1001-1010.

16 Nishiyama K, Morimoto T, Furukawa Y, et al. Chronic obstructive pulmonary disease: an independent risk factor for long-term cardiac and cardiovascular mortality in patients with ischemic heart disease. Int J Cardiol 2010; 143: $178-183$.

17 Fihn SD, Gardin JM, Abrams J, et al. 2012 ACCF/AHA/ACP/AATS/PCNA/SCAI/STS Guideline for the diagnosis and management of patients with stable ischemic heart disease: Executive Summary: A Report of the American College of Cardiology Foundation/American Heart Association Task Force on Practice Guidelines, and the American College of Physicians, American Association for Thoracic Surgery, Preventive Cardiovascular Nurses Association, Society for Cardiovascular Angiography and Interventions, and Society of Thoracic Surgeons. J Am Coll Cardiol 2012; 60: 2564-2603.

18 Diamond GA, Forrester JS. Analysis of probability as an aid in the clinical diagnosis of coronary-artery disease N Engl J Med 1979; 300: 1350-1358.

19 Montalescot G, Sechtem U, Achenbach S, et al. 2013 ESC guidelines on the management of stable coronary artery disease: the Task Force on the Management of Stable Coronary Artery Disease of the European Society of Cardiology. Eur Heart J 2013; 34: 2949-3003.

20 Patel MR, Peterson ED, Dai D, et al. Low diagnostic yield of elective coronary angiography. N Engl J Med 2010; 362: 886-895.

21 Mannino DM, Higuchi K, Yu TC, et al. Economic burden of COPD in the presence of comorbidities. Chest 2015; 148: $138-150$.

22 Hulten EA, Carbonaro S, Petrillo SP, et al. Prognostic value of cardiac computed tomography angiography: a systematic review and meta-analysis. J Am Coll Cardiol 2011; 57: 1237-1247.

23 Williams MC, Murchison JT, Edwards LD, et al. Coronary artery calcification is increased in patients with COPD and associated with increased morbidity and mortality. Thorax 2014; 69: 718-723.

24 Budoff MJ, Nasir K, Kinney GL, et al. Coronary artery and thoracic calcium on noncontrast thoracic CT scans: comparison of ungated and gated examinations in patients from the COPD Gene cohort. J Cardiovasc Comput Tomogr 2011; 5: 113-118.

25 Budoff MJ, Lutz SM, Kinney GL, et al. Coronary artery calcium on noncontrast thoracic computerized tomography scans and all-cause mortality. Circulation 2018; 138: 2437-2438. 